\title{
Actinic imaging of native and programmed defects on a full-field mask
}

\author{
I. Mochi*a K. A. Goldberg ${ }^{a}$, B. La Fontaine ${ }^{b}$, A. Tchikoulaeva ${ }^{b}$, C. Holfeld ${ }^{c}$ \\ ${ }^{a}$ Lawrence Berkeley National Laboratory, One Cyclotron Road, Berkeley, CA, 94720, USA. \\ ${ }^{b}$ Global Foundries, 1050 Arques Avenue, Sunnyvale, CA, 94085, USA \\ ${ }^{\circ}$ Global Foundries Dresden Module One. KG Wilschdorfer Landstr. 101, 01109 Dresden, Germany.
}

\begin{abstract}
We describe the imaging and characterization of native defects on a full field extreme ultraviolet (EUV) mask, using several reticle and wafer inspection modes. Mask defect images recorded with the SEMATECH Berkeley Actinic Inspection Tool (AIT), an EUV-wavelength $(13.4 \mathrm{~nm})$ actinic microscope, are compared with mask and printed-wafer images collected with scanning electron microscopy (SEM) and deep ultraviolet (DUV) inspection tools.

We observed that defects that appear to be opaque in the SEM can be highly transparent to EUV light, and inversely, defects that are mostly transparent to the SEM can be highly opaque to EUV. The nature and composition of these defects, whether they appear on the top surface, within the multilayer coating, or on the substrate as buried bumps or pits, influences both their significance when printed, and their detectability with the available techniques. Actinic inspection quantitatively predicts the characteristics of printed defect images in ways that may not be possible with nonEUV techniques.
\end{abstract}

As a quantitative example, we investigate the main structural characteristics of a buried pit defect based on EUV through-focus imaging.

Keywords: Mask inspection, defects, extreme ultraviolet, EUV, actinic, lithography, microscopy

\section{INTRODUCTION}

Mask defectivity is one of the main issues for advanced lithography in any future technology node. This is especially true for EUV lithography where the mask, the absorber pattern, and the various types of defects can have wavelengthspecific optical properties. Developing reliable and accurate methods for defect inspection and imaging is an essential step towards the deployment of EUV lithography.

While DUV microscopy and SEM provide valuable information at high resolutions, their sensitivity to defects can be remarkably different from EUV-wavelength imaging, and they cannot guarantee measurements that predict EUV printing performance. Our research shows that some defects that are strongly evident in an SEM will not actually print, while others, which are faintly detected with SEM or DUV inspection, can clearly appear on the wafer. Aside from printing in photoresist, EUV aerial imaging is the only technique that provides quantitative information on the interaction between the EUV light and the blank or patterned mask surface.

Following exposure in the ASML Alpha Demo Tool (ADT) at CNSE in Albany [1], New York, the reticle and its printed wafers were inspected using SEM and two KLA tools: the 2800 Series broadband and brightfield DUV/UV/VIS inspection platform and the TeraScanHR 587 reticle defect inspection system [2]. A classification system presented previously [3] separated the defects into the following categories: cleaning residue, particle, pattern defect, blank defects, and nuisance defects.

In this paper we describe the imaging of native defects on a EUV mask from Global Foundries, carried out using the AIT [4], an EUV Fresnel zoneplate microscope dedicated to photomask research [5]. EUV imaging, performed with the AIT, adds new information about the optical properties of these reticle defects. Our goal was to investigate the relationship between the appearance of various defects in the different defect-imaging tools. Our observations show that it is difficult to predict the EUV response from the SEM or DUV appearance.

By studying the through focus evolution of a defect's aerial image, with quantitative comparison to simulated defect images, it is possible to investigate physical characteristics (such as the apparent defect height and three-dimensional 
profile) and to discriminate between amplitude, phase, and complex defects. Experimentally, we observe both native phase and amplitude defects, yet many of the defects that we have investigated show behaviors characteristic of both types.

\subsection{The SEMIATECH Berkeley Actinic Inspection Tool (AIT)}

The AIT is a synchrotron beamline-based, EUV-wavelength, Fresnel zoneplate microscope that records highly magnified images of the reflective mask surface. The AIT is located at the Advanced Light Source (ALS) at Lawrence Berkeley National Laboratory (LBNL). In the last several years, the AIT has been significantly upgraded to provide a high degree of imaging flexibility, with variable resolution and magnification. The microscope operates at a central wavelength of $13.4 \mathrm{~nm}$, with narrow-band illumination $(E / \Delta E=1450)$. It is equipped with five zoneplates with different resolution and magnification values. Recently we installed a two-dimensional scanning illumination system to have a more uniformly illuminated field of view. We have also developed techniques to improve the zoneplate alignment to obtain diffraction limited imaging over a 5-8- $\mu \mathrm{m}$ region of interest within the field of view [6].

The measurements described in this paper were carried out using a zoneplate objective lens with a 750- $\mu \mathrm{m}$ focal length, a magnification of $907 \times$ and a mask-side numerical aperture of $0.0875(0.35-\mathrm{NA}, 4 \times$ wafer-side equivalent). We note that this resolution is $40 \%$ higher than the current generation of EUV alpha tools: at $0.25-\mathrm{NA}$, the ADT's mask side NA is 0.0625 [7].

\subsection{KLA Tencor inspection tools}

The 2800 Series is a broadband DUV/UV/VIS patterned wafer inspection platform for sub-65-nm design rule development and production. It provides $2 \times$ better throughput than KLA's previous-generation DUV tool and features a roadmap that extends the platform to design rules of $45-\mathrm{nm}$ and beyond. It has an array of optical modes to provide an optimal sensitivity level for particular layers, designs, and process types [8]

The TeraScanHR 587 Reticle Defect Inspection System includes high-resolution optical imaging, capabilities for inspecting aggressive Optical Proximity Correction (OPC), and high speed image processing. These new advances in technology enable the TeraScan HR reticle defect inspection system to find all types of reticle defects including random reticle manufacturing defects such as oversize and undersize critical dimensions and Sub-Resolution Assist Feature (SRAF) defects, and extensions [9].

\section{MEASUREMENTS DESCRIPTION}

The measurements described here were recorded on a full-field, 45-nm test chip reticle, the Typhoon Mask. The reticle is a Schott-Lithotec blank substrate with a silicon capped multilayer coating. Over the multilayer there are a $10-\mathrm{nm} \mathrm{SiO}_{2}$ buffer layer and a patterned TaN absorber layer with anti-reflecting coating optimized for the wavelength of the KLA mask inspection tool $(275 \mathrm{~nm})$.

Reticle exposures were performed at the College of Nanoscale Science and Engineering (CNSE) in Albany, New York, using the ASML Alpha Demo Tool, an EUV, 0.25 NA, full-field, step and scan system. The rest of the processing was carried out at AMD's Fab36, with the exception of the EUV inspection performed at LBNL.

The defects were originally identified using the various non-EUV techniques. The exposed wafers were inspected with a KLA-Tencor 2800, and SEM micrographs of repeater defects were collected. The reticle was reviewed using an SEM, the KLA-Tencor 587 at DUV wavelength, and the AIT.

We inspected areas of the reticle where known defects had previously been discovered. Mask navigation in the AIT is complicated by its limited field of view and non-kinematic mask loading system. Nonetheless, all of the defects selected for AIT evaluation were found and were observable in the EUV images. From this mask, twenty-nine defects were studied in the AIT.

The through-focus properties of patterns (and especially, defective patterns) determine the width of the process window parameter space. Through-focus measurement is the most common mode of data collection for the AIT. Originally, the AIT used longitudinal (focus direction) mask motion to acquire a through-focus image series. Currently, the preferred and most stable mode of through-focus data collection is to use tiny steps in wavelength, exploiting the inherent chromatic focal dependence of the zoneplate lens. This technique is described in Ref. [10]. 
For each inspection point, we acquired a series of images through focus. With step size $0.8 \mu \mathrm{m}$ ( $50 \mathrm{~nm}$ in $4 \times$ wafer units) and in a range of $6.4 \mu \mathrm{m}$ ( $400 \mathrm{~nm}$ in wafer units). In addition to finding the best focus plane, through-focus imaging allows us to study the phase characteristics of each defect, as described below.

The AIT requires fine alignment at each measurement point. At best alignment, the diffraction-limited sweet spot of the image covers a circular area, approximately $6 \mu \mathrm{m}$ in diameter [6], while the entire field of view is nearly $30 \mu \mathrm{m}$ across. Most of the defects we inspect have a linear size of less than one micron and fit within a fraction of the sweet spot area.

\subsection{Amplitude and phase defects}

It has become conventional to characterize EUV mask defects as amplitude defects or phase defects. We observe that many defects have properties of both, and can thus be characterized as complex defects, for their effect on the reflected field amplitude.

Amplitude defects perturb the reticle's pattern by reducing the local reflectivity of the mask. They can be caused by particles or contamination on the top surface of the multilayer, or they can result from of imperfections, oxidation, or damage within or below the multilayer coating. Our observations show that while defects may be visible in SEM review, SEM alone cannot predict the effect that given defects will have on the printed wafer.

In the case of amplitude-only defects, the aerial images show a symmetric blurring on both sides of focus. This effect can be seen in Fig. 1. Here we simulate the behavior of a $1-\mu \mathrm{m}$-long dark defect with a Gaussian reflectivity profile and 125-nm full-width at half-maximum (FWHM). The defect becomes opaque at its center. To approximately replicate imaging in the AIT, a coherently illuminated mask and a 0.0875 -NA objective lens (0.35-NA, 4x wafer-side equivalent) were used in the calculation.

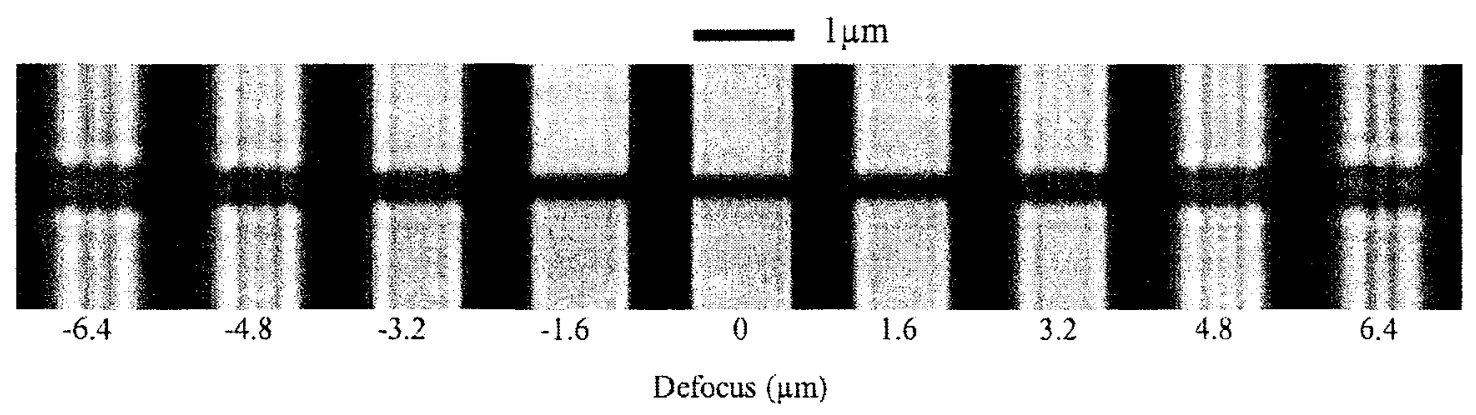

Figure 1. Through-focus imaging simulation of an amplitude defect with Gaussian profile. The symmetric through-focus behavior is characteristic of amplitude-only defects. These steps correspond to $100-\mathrm{nm}$ wafer-side steps in a $4 \times$ system.

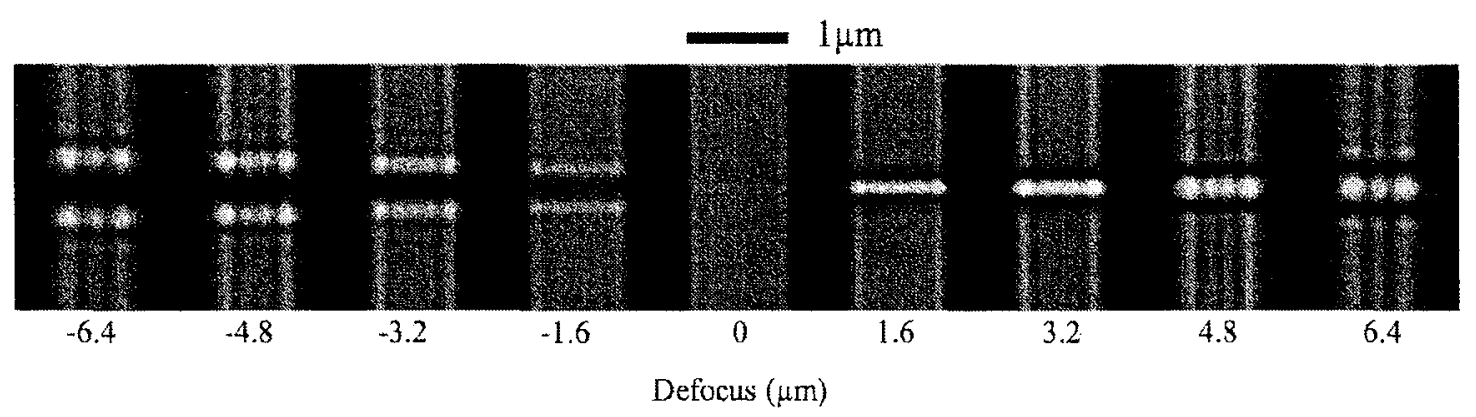

Figure 2. Through-focus simulation of a pit phase defect across a l- $\mu \mathrm{m}$ line, with a $125-\mathrm{nm}$ FWHM Gaussian phase profile that reaches a maximum value of $\pi / 2$. The asymmetric intensity behavior through focus is characteristic of a phase defect. These steps correspond to 100 -nm wafer-side steps in a $4 \times$ system. Here the intensity reaches a maximum normalized amplitude of $290 \%, 3.2 \mu \mathrm{m}$ above focus ( $200 \mathrm{~nm}$ in wafer scale).

Phase defects induce a local phase variation of the light reflected from the mask surface. This phase shift can be caused by particles buried in or below the multilayer (i.e. bump defects) or from holes or voids in the substrate (i.e. pit defects). 
The characteristic through-focus behavior of phase defects is an asymmetric intensity profile on either side of best focus. Figure 2 contains a phase-defect simulation similar to Fig. I, except that the amplitude remains constant across the defect, while the phase varies in a Gaussian profile with a peak value of $\pi / 2$. At a wavelength of $13.4 \mathrm{~nm}$ this is equivalent to a maximum optical path difference of $3.35 \mathrm{~nm}$, corresponding to a defect surface depth of $1.68 \mathrm{~nm}$.

\section{EXPERIMENTAL MEASUREMENTS}

More than twenty defects on the test mask were investigated with the AIT. The four examples presented in this section were chosen to reveal the variety of different defect inspection results we have found.

\subsection{A structured defect highly opaque to EUV light}

Figure 3 shows an image of the same defect recorded with four different measurement techniques. The SEM image reveals the presence of a structured defect approximately $2 \mu \mathrm{m}$ long, with a dark core and a pale, semi-transparent contour. The appearance of the defect is significantly different in the DUV image, which shows no appreciable difference between the absorption of the dark core and the semi-transparent halo around it. The AIT image in Fig. 3B clearly shows the whole contour of the defect, matching the SEM image of the printed wafer.

Looking closely at the AIT image (Fig. 3B), we observe that the two opposite corners of the lower-right bright rectangle partially shadowed by the defect are still visible at reduced intensity, although they did not print (Fig. 3D). This discrepancy between the two images may be due in part to the higher resolution and numerical aperture of the AIT and the ADT used in these measurements $(0.35$ versus 0.25 ). The finite photoresist resolution and decreased peak intensity of these bright corners may also contribute to their disappearance from the printed wafer image. The symmetric intensity behavior through focus suggests that the defect in question is an amplitude defect, laying above or etched into the multilayer surface.
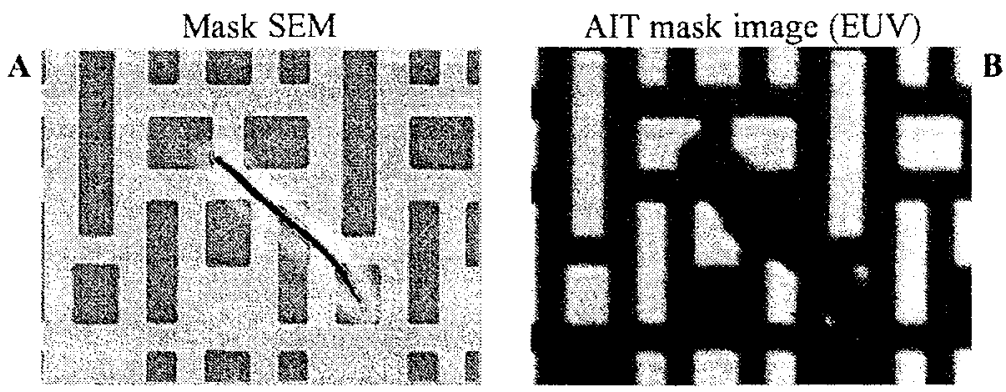

KLA 587 mask image (DUV)
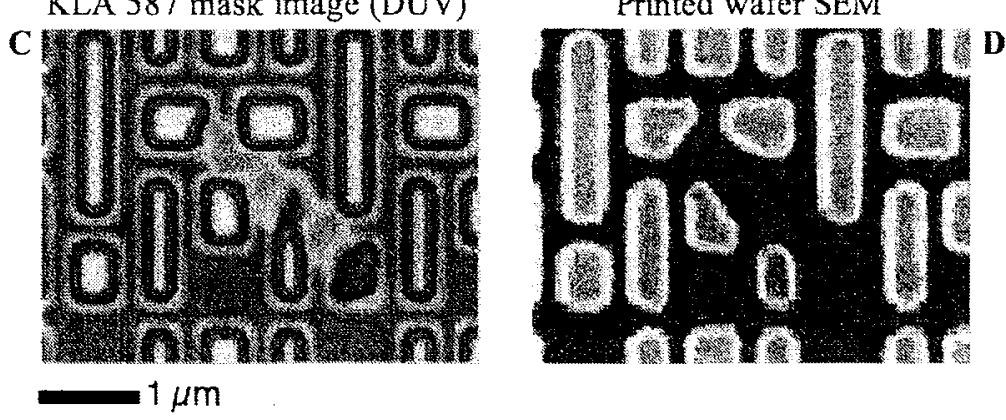

Figure 3. Visual comparison of an EUV-opaque defect imaged with mask and printed-wafer SEM, the AIT and the KLA 587.

\subsection{An EUV transparent defect}

Another example of a structured amplitude defect is shown in Fig. 4. The SEM reveals a large "stain," contamination over the pattern with an irregular contour and approximately $1 \mu \mathrm{m}$ radius. The appearance of the defect in the SEM 
micrograph suggests that this stain could yield a major contrast loss. The DLV review also shows a strong intensity variation with a modification in the pattern contour, close to the corner of the line. However, the AIT image, instead reveals that most of this defect is highly transparent to EUV light, and does not induce a significant reflectivity loss. The only portion of the defect that creates an intensity loss sufficient to print is the lower right edge of the stain which actually interrupts the line. Although this interruption was visible in the printed wafer, it was electrically irrelevant [3].
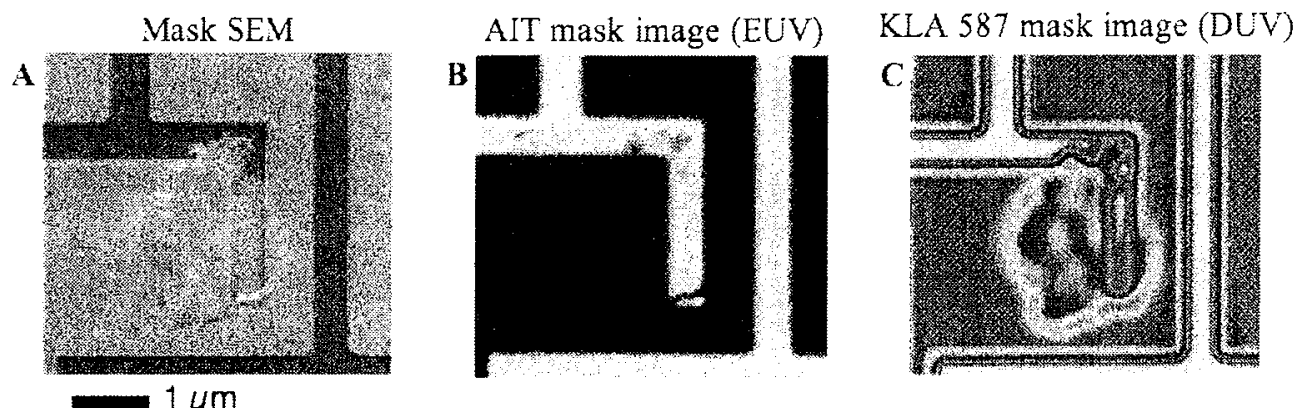

Figure 4. Visual comparison of a semitransparent EUV defect inspected with the SEM, the AIT and the KLA 587.

\subsection{A small amplitude defect}

Figure 5 shows images of an amplitude defect that is clearly detected by the SEM and the AIT. The DUV mask image shows a subtle, asymmetric rounding of the lower right corner of the rectangle--no quantitative information about the effective defect size or edge contour displacement was available to us. The pattern contour seen in the AIT image closely matches the reticle's SEM review and the printed wafer image, indicating that this small defect is highly opaque to EUV light.
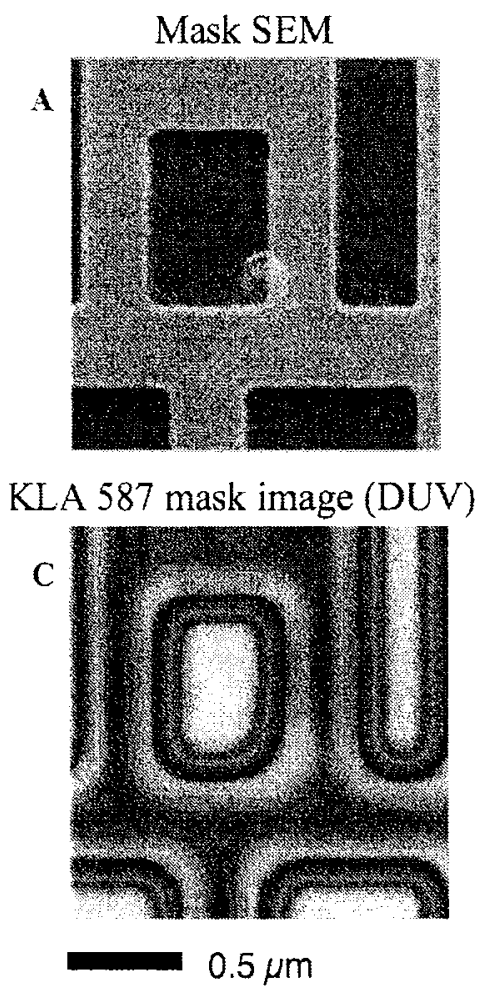
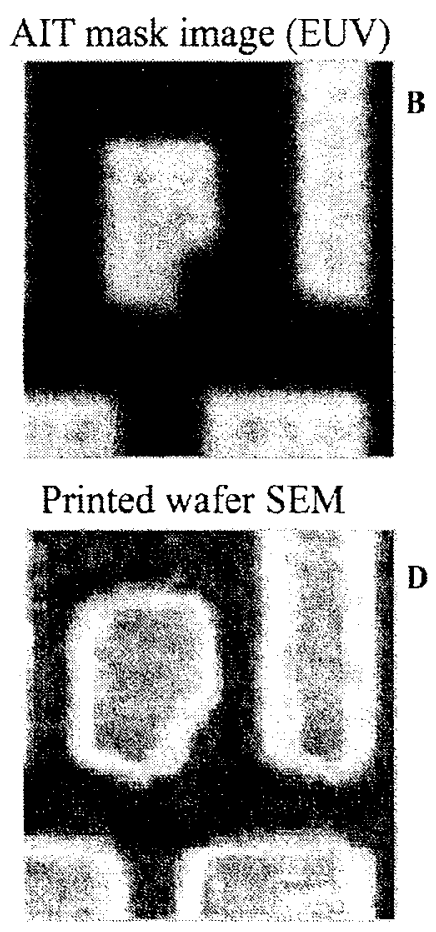

Figure 5. Visual comparison of an amplitude defect inspected with the SEM. the AIT and the KLA 587. In the lower right panel the SEM image of a printed wafer is shown 


\subsection{ELV aerial images and simulation of a complex defect}

Phase defects can be difficult to observe with an SEM or under DUV illumination. A buried defect's topography could be flattened and smoothed by the multilayer and yet still significantly modify the reflected EUV field. We show here how a phase defect can be observed and characterized with EUV inspection.

Figure 6 shows an interesting case of pit phase defect with a strong EUV response and a characteristic behavior of a complex defect. Here, the SEM image reveals a low contrast vertical trench across a $1-\mu \mathrm{m}$ linc. The DLV image confirms the presence of a vertical defect, but with little contrast. (Quantitative information is not available.)

The AIT inspection instead shows that the line is completley interrupted. This result is confirmed by the printed wafer SEM. This defect was also inspected with an atomic force microscope (AFM), which revealed a trench in the line with a roughly Gaussian shape in one dimension, with an average depth of $20 \mathrm{~nm}$ and a FWH.M $125 \mathrm{~nm}$.

The AIT through-focus image series of this defect shows the asymmetric intensity behavior characteristic of a pit phase defect (see Fig. 7). In simulation, we were able to reproduce the characteristics of this defect to approximately quantify the its effective phase and amplitude modification. To simplify the reconstruction we isolated the central part of the defect, cropping a rectangular detail area of $300 \times 200 \mathrm{~nm}$ (with the long direction horizontal, perpendicular to the defect's vertical orientation). By averaging the rows vertically into a single intensity profile, we reduce this particular analysis to a one-dimensional profile reconstruction. However, the full two-dimension behavior of the defect, and its interaction with the surrounding pattern, can be studied. We performed this operation on each image in the through-focus scries and to create an array of defect profiles at the different focal positions.
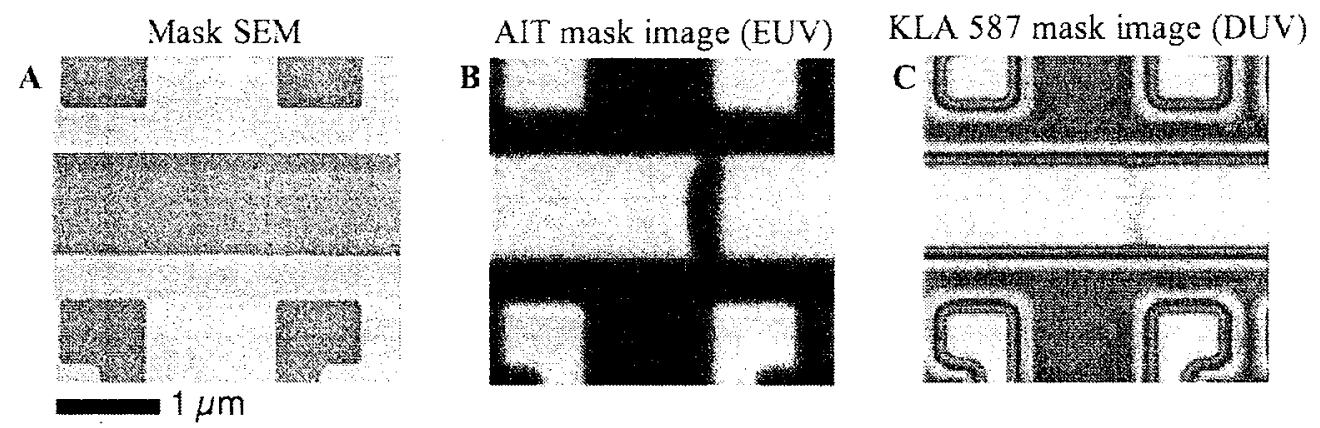

Figure 6. Visual comparison of a complex defect inspected with the SEM, the AIT and the KLA 587. The defect appears very faint in the SEM image in the right panel. Thin vertical lines on the SEM micrograph are artifacts of the SEM inspection.

The defect was modeled as a buried trench in the multilayer substrate, with a Gaussian-shaped profile. Empirically, we found that the most effective model for describing this defect contained a complex phase and amplitude profile. The electric field reflected by the defect was modeled as:

$$
E(x)=A(x) \exp [i \phi(x)] .
$$

Where $A(x)$ and $\phi(x)$ are the defect reflected amplitude and phase profiles respectively and their expression is given by:

$$
A(x)=1-a \exp \left[-\left(x-x_{0}\right)^{2} / 2 \sigma^{2}\right], \quad \phi(x)=p \exp \left[-\left(x-x_{0}\right)^{2} / 2 \sigma^{2}\right] .
$$

The fraction of the amplitude absorbed by the defect is given by the real-valued parameter $a$ (bounded on $[0,1]$ ) and the maximum phase shift is $p$. Images were calculated from the electric field profile $E(x)$ using a Fresnel trasform and modeling the imaging system as a single lens, with a circular pupil, 0.0875 mask-side $\mathrm{NA}$, and $907 \times$ magnification ratio. We repeated the calculation through focus, changing the object conjugate plane in steps of $0.8 \mu \mathrm{m}$ to match the AIT data.

To fit the measured through-focus array of intensity profiles, we used a simple merit function: the variance of the difference between the measured and the simulated intensity profiles: 


$$
M(\mathbf{v})=\sum_{i=1}^{n} \sum_{i}\left[I_{i}(x, \mathbf{v})-\hat{I},(x)\right]^{2}
$$

Where $\mathbf{v}$ is a the vector of the fit parameters, $n$ is the number of images in the series, $I,(x . v)$ and $\hat{l}(x)$ are the simulated and measured profile intensities in the $j^{\text {th }}$ focal plane image.

We fit the free parameters using a non-linear least squares routine with an initial guess of $\sigma$ and $p$ based on the shape of the trench measured by the AFM, and an initial value of $a$ set equal to zero (i.e. a perfect phase defect).

The estimated peak phase of the defect was $p=7.0 \pi$, corresponding to a defect depth of $23.45 \mathrm{~nm}$. The estimated Gaussian sigma was $\sigma=54.1 \mathrm{~nm}$, corresponding to a defect FWHM of $127.3 \mathrm{~nm}$ and the estimated peak absorption was $a=0.8$. The resulting values of the parameters $a, p$ and $\sigma$ were used to generate the two dimensional representation of the defect and line shown in Fig. 7.

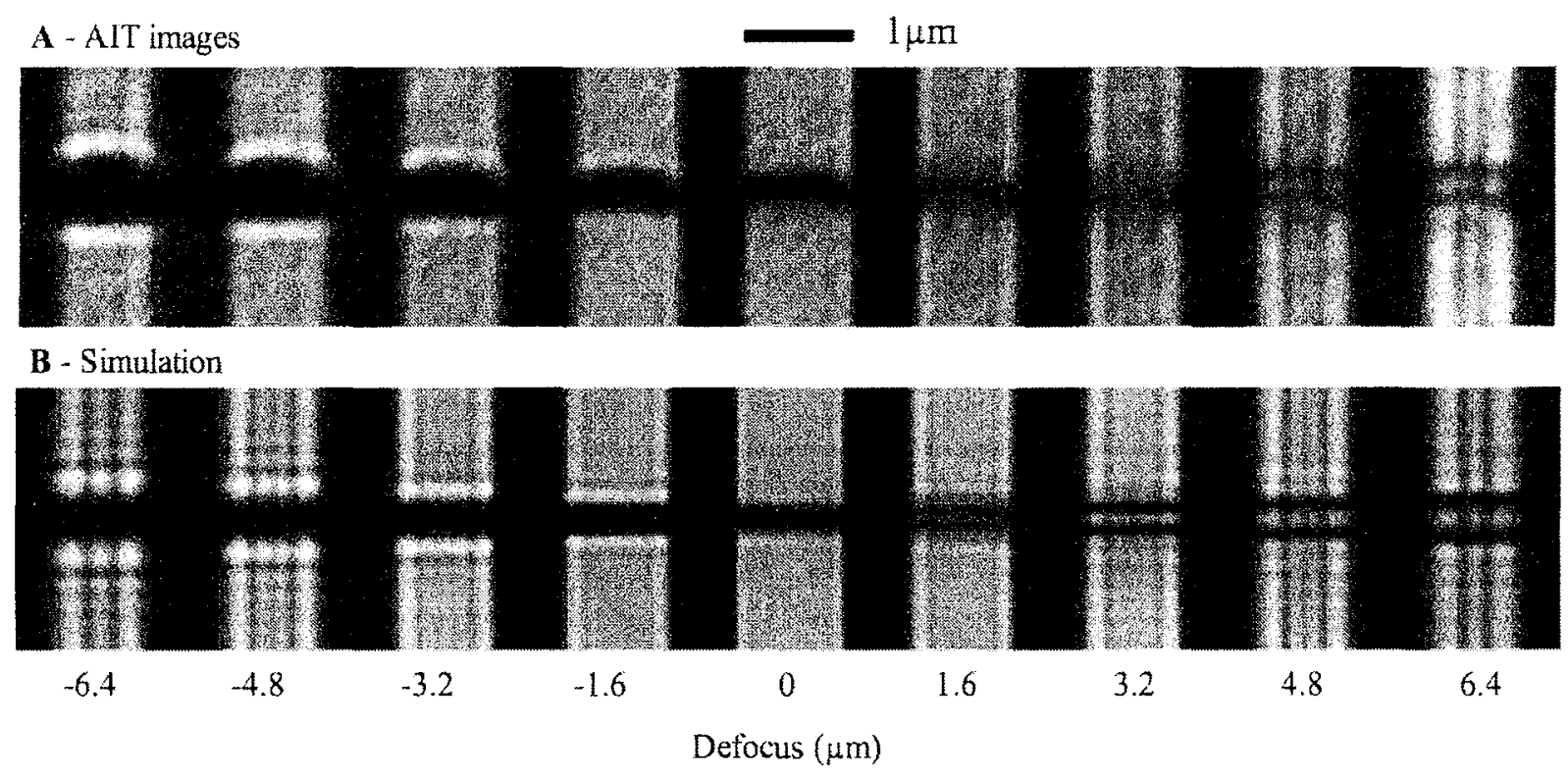

Figure 7 (A) shows the through-focus image series of the pit phase defect of Fig. 6, rotated by $90^{\circ}$, and (B) a reconstruction from best fit parameters: $p=7.0 \pi, a=0.8$. For brevity we are showing every other image in the series. The characteristic through focus behavior of phase defects is visible: an asymmetric intensity at the defect center.

This reconstruction is based on one single dataset, and the fitting parameter uncertainties are not well quantified. However, the fit converges to values that are in good agreement with the AFM measurements, and match visually with the AIT image data.

More measurements and simulation will allow to characterize the sensitivity and the limitations of this approach. Further development of this technique will include the definition of a more sensitive merit function and careful choice of the optimization algorithm. This could be done cross-comparing our results with electro-magnetic simulation software. We believe that this is an important first step towards the development of a quick and reliable technique to characterize phase and amplitude defects on EUV masks.

\section{CONCLUSION}

Due to the wavelength-specific response of EUV mask materials and defects, images recorded with EUV microscopy and photoresist images can differ substantially from SEM and DUV measurements. The importance of gathering defect 
cross-correlation data between the different inspection modes is heightened by the current unavailability of highthroughput EUV mask imaging tools.

The small number of defects that have been reviewed in multiple tools must be examined carefully to extract as much information as possible. While it is difficult to generalize or predict the ultimate success of DUV versus EUV highvolume inspection tools, it is clear from the measurements presented here, that substantial qualitative differences in defect appearance and severity among the different imaging tools are the norm, and not the exception. One limitation of the current measurements is that all of the defects investigated in this small study were originally detected with DUV inspection tools. This fact leaves open the question of EUV lithography's vulnerability to so-called actinic-only defects, detectable only with ELV light. One cannot predict the advances in sensitivity that may improve the small-defect capture rate of the DLV tools, but we believe that over time, the shrinking pattern design rules inevitably point toward the necessity of ELV imaging.

The measurements presented here illustrate important aspects of the limited available cross-correlation data, collected from the same mask defects, with different imaging technologies: EUV $(13.4 \mathrm{~nm})$, DUV $(257 \mathrm{~nm})$, SEM, and AFM. The data show that extreme ultraviolet microscopy of EUV reticles provides a faithful, predictive representation of the aerial image detected in photoresist imaging.

Using limited available data, we presented qualitative comparisons among images collected with the EUV-wavelength SEMATECH Berkeley Actinic Inspection Tool (AIT), SEM images of the reticle and of wafers printed by the ASML Alpha Demo Tool (ADT) at CNSE, DUV microscopy of the mask using a KLA-Tencor TeraScanHR 587, and DUV microscopy of the printed wafers, using a KLA 2800 Series wafer-inspection tool.

The comparisons showed that SEM and DUV review of mask defects can overestimate or underestimate the intensity variations and apparent size of defects as seen with EUV light. The severity of amplitude defects is difficult to predict from non-EUV imaging. Phase defects, on the other hand, can originate in the lower levels of the multilayer or the substrate; they can appear under EUV illumination even if the top surface of the mask is relatively flat. Analysis shows that EUV light penetrates with appreciable amplitude to the bottom layer of a multilayer coating, and therefore, the reflected field is sensitive to the shape of every layer. As a result, while the SEM and DUV are essential and invaluable tools to assess the physical nature mask defects, they alone may be inadequate to predict the printed pattern in the presence of defects.

Our analysis shows that defect modeling makes it possible to evaluate the effective physical properties of mask defects. We studied the through focus evolution of aerial images and extracted convincing quantitative information about the characteristics of phase defects as they appear under EUV illumination. With more cross-corrclation data as input, these studies should be expanded to derive general methods for EUV defect characterization. Such work would increase our understanding of the critical defect threshold, and could serve to improve the predictive powers of SEM and DUV inspection tools.

\section{ACKNOWLEDGEMENTS}

We wish to acknowledge the support of SEMATECH project leader Sungmin (Sean) Huh. This work was supported by SEMATECH, through the L.S. Department of Energy under Contract No. DE-AC02-05CH11231.

\section{REFERENCES}

[1] K. Cummings, T. Laursen, B. Pierson, S. Han, R. Watso, Y. van Dommelen, B. Lee, Y. Deng, B. La Fontaine, T. Wallow, U. Okoroanyanwu, O. Wood, A. Tchikoulaeva, C. Holfeld, J. Peters, C. Koay, K. Petrillo, T. DiBiase, S. Kini, H. Mizuno, "An Investigation of EUV Lithography Defectivity," Proc. SPIE 7122, 71222G (2008).

[2] A. Tchikoulaeva, et al. "A Practical Approach to EUV Reticle Inspection," International Symposium on Extreme Clitraviolet Lithography, Sep.28-Oct. 1, (2008), Lake Tahoe, USA

[3] A. Tchikoulaeva, U. Okoroanyanwu, O. Wood, B. La Fontaine, C. Holfeld, S. Kini, M. Peikert, C. Boye, C.-S Koay, K. Petrillo, H. Mizuno. "EUVL Reticle Defectivity Evaluation," Proc. SPIE 7271, 727117 (2009). 
[4] K. A. Goldberg, P. P. Naulleau, A. Barty, S. B. Rekawa, C. D. Kemp, R. F. Gunion, F. Salmassi, E. M. Gullikson,E. H. Anderson, H.-S. Han, "Performance of actinic ELVL mask imaging using a zoneplate microscope," Proc. SPIE 6730, 67305E-1-12(2007).

[5] K. A. Goldberg, I. Mochi, P. P. Naulleau, H.-S. Han, S. Huh, "Benchmarking ELV mask inspection beyond 0.25 NA," Proc. SPIE 7122, $71222 \mathrm{E}-1$ (2008).

[6] I. Mochi, K. A. Goldberg, P. P. Naulleau, S. Huh, "Improving the performance of the Actinic Inspection Tool with an optimized alignment procedure," Proc. SPIE 7271, 727123, (2009).

[7] J. V. Hermans, B. Baudemprez, G. Lorusso, E. Hendrickx, A. van Dijk, R. Jonckheere, and A.-M. Goethals, "Stability and imaging of the ASML EUV alpha demo tool," Proc: SPIE 7271, 72710T (2009).

[8] hetp: www kla-tencor.comicertified-used-cquipment:2800series.heml

[9] http: www kla-tencorcom reticle-manufacturing terascanhr.html

[10] K. A. Goldberg, I. Mochi, S. Huh, "Collecting EUV mask images through focus by wavelength tuning," Proc. SPIE $7271,72713 \mathrm{~N}-1-8(2009)$. 